\title{
UNDERSTANDING THE CHALLENGE OF CYBERSECURITY IN CRITICAL INFRASTRUCTURE SECTORS
}

\author{
Maurice DAWSON \\ Illinois Institute of Technology, Chicago, United States of America \\ mdawson2@iit.edu \\ Robert BACIUS \\ Illinois Institute of Technology, Chicago, United States of America \\ rbacius@hawk.iit.edu \\ Luis Borges GOUVEIA \\ University Fernando Pessoa, Porto, Portugal \\ lmbg@ufp.edu.pt \\ Andreas VASSILAKOS \\ Illinois Institute of Technology, Chicago, United States of America \\ avassilakos@hawk.iit.edu
}

\begin{abstract}
The cybersecurity of critical infrastructures is an essential topic within national and international security as 16 critical infrastructure sectors touch various aspects of American society. Because the failure to provide adequate cybersecurity controls within the critical infrastructure sectors renders the country open to an attack that could have a debilitating effect on security, national public health, safety, and economic security, this matter is so vital that there is the Presidential Policy Directive (PPD) 21 Critical Infrastructure Security and Resilience advances a national policy to strengthen and maintain secure, functioning and resilient critical infrastructure. An organization identified as the Cybersecurity and Infrastructure Security Agency (CISA) at the Department of Homeland Security (DHS) has the mission to be the risk advisor for the United States (US). Other organizations, such as the National Security Agency (NSA), have approved a specific Knowledge Unit (KU) to address cybersecurity for critical infrastructures associated with doctoral-level granting programs. To address this challenge, it is necessary to identify threats better and defend against them while mitigating risks to an acceptable level. Only then can a nation build a more secure and resilient infrastructure for the future while defending against present-day bad actors as cyberwarfare, cyber espionage, and cybersecurity attacks are the modern-day threats that need to be addressed in planning, designing, implementation, and maintenance. Therefore, the researchers developed a case study reviewing threats against different sectors defined in the PPD.
\end{abstract}

KEYWORDS: critical infrastructures, hackers, cybersecurity policy, cybersecurity controls, national security

\section{Introduction}

Cyberwarfare is the use of technology to cause nation-scale negative impacts and damage. This can be seen in forms including computer viruses targeting various forms of infrastructure, denial of service attacks to halt vital technology services, digital propaganda to shift political belief, cyber espionage to gain classified information, and malware in attempts to destroy technological systems. These types of attacks are aimed to create disasters that embody actual war. Several high profile attacks on critical infrastructure 
show us how vulnerable a nation can be. The government of the United States of America has identified the plethora of risks associated with terrorism in the cyber realm and its potential consequences. Cyberwarfare's presence exceeds systems in the computer grid. It also includes any system that can be vulnerable; mobile devices, embedded systems, and those connected wirelessly are examples. Lewis (2002) states that cybersecurity literature assumes that critical infrastructures and computer networks' associated vulnerabilities are the same.

DHS is concerned with cyberattacks on critical infrastructure sectors, for example, supervisory control and data acquisition (SCADA) systems. SCADA systems are liable for monitoring and adjust switching among other processes within critical infrastructure sectors in an autonomous manner. DHS is concerned about SCADA systems as they are frequently unmanned and remotely accessed. As they are remotely accessed, anyone can take control of assets to critical infrastructure remotely. There has been increasing mandates and directives to ensure any system deployed meets stringent requirements. As the Stuxnet worm has become a reality, future attacks could be malicious code directly targeting specific critical infrastructure locations. Once an attack has been successfully carried out, remnants of the code are found online in the public domain and later on the Dark Web. This is a problem as script kiddies could repurpose this code for a new target.

\section{Time of Events}

Throughout the fall of 2010, numerous news outlets pronounced that Stuxnet was a game-changer in digital warfare (Denning, 2012). Stuxnet was unpredictable and originally was intended to target just one specific system. Also, this nefarious program could recognize the area and system type, among other information. Furthermore, this worm had the functionality to attack a specific system if it could identify parameters that were incorporated in its software code. Stuxnet's impact resulted in tampering with software in a programmable logic controller (PLC) responsible for centrifuges located at Natanz. This tampering ultimately disrupted Iran's nuclear program.

Flame was another example where a highly sophisticated malicious program was being used as a cyber weapon in various countries. Kaspersky discovered Flame, and identified it as the most massive weapon among the ones under the same category. Flame was developed to conduct espionage by breaching information related to systems, stored files, contact data, and audio conversations (Munro, 2012). Flame was conducted as a series of attacks against Iran's oil industry. Attacking a nation's critical infrastructure and resources are militarystrategic tactics that have been undertaken successfully via thousands of code lines.

With that in mind, the U.S. government has made a few efforts to manage cyber breaches and establish security controls. For instance, under President Obama's leadership, the Executive Order - Improving Critical Infrastructure Cybersecurity was given (Obama, 2013). This Order accommodates, in addition to other things, data sharing among government and private organizations, to incorporate a spread of reports in regards to critical infrastructure resources, civil liberty protection, critical infrastructure risk reduction, cyber-security framework development, voluntary cybersecurity program creation, and critical infrastructure cybersecurity risk identification (Obama, 2013).

On February 12, 2014, mandated by President Obama's Executive Order, the "Framework", with the extended title "Framework for Improving Critical Infrastructure Cybersecurity, version 1.0", was developed and issued. The 41 pages of the Framework pertained to issues as described in the contents of the Executive Order (National Institute of Standards and 
Technology, 2014). Because of the fact that digital threats are persistently evolving, the Framework would need to continually be updated as well; essentially it has to stay as a living document (NIST, 2014). The framework's core components are comprised of Functions, Categories, Subcategories, and Informative References (NIST, 2014). The framework's core functions are to Identify, Protect, Detect, Respond, and Recover (NIST, 2014).

The Department of Health and Human Services (HHS) and the Department of Homeland Security (DHS) were required to report information regarding their specific sectors of critical infrastructure ("the Reports") (The White House, 2014).

The DHS report comprised three (3) areas:

- chemical facility antiterrorism standards;

- maritime critical infrastructure cybersecurity standards;

- transportation critical infrastructure cyber-security standards (U.S. Department of Homeland Security, 2014).

The EPA report focused on the standards regarding cybersecurity for the water and wastewater critical infrastructure sectors. The HHS report focused on the standards regarding cybersecurity for the food and drug critical infrastructure sectors. It also covered exercises specially developed to prepare for the mitigation of attacks targeting medical devices in healthcare (U.S. Department of Health and Human Services, 2014).

The United States federal government is proud of the work towards safeguarding the cybersecurity of critical infrastructure sectors and recognizes the need for further work (The White House, 2014). While The Order, the Framework, and the Reports are major steps towards a cyber-security national policy, they are restricted to those areas defined as critical infrastructure. Critical infrastructure, by definition, includes industries and areas, both physical and virtual, that are "...so vital to the United States that the incapacity or destruction of such systems and assets would have a debilitating impact on security, national economic security, national public health or safety, or any combination of those matters..." (Obama, 2013). It is important to note that smartphones, tablets, or other mobile devices might not fall into that category. Furthermore, even in the case of critical infrastructure, the Order excludes explicitly any essential regulation of infrastructure security outside the scope of the current law, meaning that it only requires specific actions to be taken but does not provide for a more significant policy or legal protection than what is already covered by other laws. Also, it is an Executive Order and applies exclusively to the federal agencies under the government's control's executive branch anyway (Obama, 2013).

\section{Challenges}

With recent events from global pandemics to the shift in economic powers, many challenges lay before us. These challenges created new adversaries and motivated older ones to get re-engaged. Understanding these challenges will help develop a mitigation plan to avoid these severe consequences by failing to do something. Table no. 1 shows the threat and gives an example of what that threat is. This table provides insight at a high level of what organizations will need to be aware of when deploying systems that may be inadequately secured.

Motivated hackers, hacking groups, and nation-states aim to sabotage infrastructure systems by changing or adding code in such systems to negatively manipulate functions with the goal of information leakage, total system failure, or system harm (Wilson, 2014). An example of this could be a hacker injecting malware into a power grid system. With this 
unauthorized control, the hacker can manipulate electrical power functions or possibly shut the grid down. There are many dependencies when it comes to critical infrastructure working as expected. If an attack happens on one system, it disrupts multiple other networks (Robles, Choi, Cho, Kim, Park, \& Lee, 2008).

An example of this is initially shutting down a traffic control system to disrupt vehicle traffic flow and creating a constant traffic control system, such as keeping all traffic lights green to increase traffic violations. Cyber attacks on water supply infrastructure systems can be devastating, both directly and indirectly (Lewis, 2002). Presently, if hackers can gain unauthorized control of a watergate system, they can cause flooding resulting in damages to property and lives. Indirectly, effects can lead to sanitation and purification issues for neighborhoods and cities or even deny access to water for firefighters in emergency events. Cyber attacks on electrical infrastructure systems can occur when a hacker gains unauthorized control of an electrical power system to cause nearby blackouts/brownouts resulting in limited access to the internet (Lewis, 2002). This can carry over into other effects such as shutting down traffic lights to cause traffic complications and limiting public communication channels as WiFi connection to routers that use electricity can be shut down without a proper backup electrical source.

Table no. 1

Threats

\begin{tabular}{|c|c|c|c|c|}
\hline Threat & Damage to Occur & Example & Keywords & Reference \\
\hline $\begin{array}{l}\text { Cyber-Physical } \\
\text { system break-ins }\end{array}$ & $\begin{array}{l}\text { Compromising automated } \\
\text { manufacturing processes leading to a } \\
\text { halt in production or employee safety } \\
\text { risks. }\end{array}$ & $\begin{array}{l}\text { A hacker is gaining access to a } \\
\text { manufacturing system that utilizes } \\
\text { physical feedback technology, such as } \\
\text { sensors, to track manufacturing output. } \\
\text { Once access is achieved, the hacker can } \\
\text { stop system processes such as } \\
\text { manufacturing output. }\end{array}$ & $\begin{array}{l}\text { Cyber-physical } \\
\text { system }\end{array}$ & $\begin{array}{l}\text { (Bracho, Saygin, Wan, } \\
\text { Lee, \& Zarreh, 2018). }\end{array}$ \\
\hline $\begin{array}{l}\text { Process parameter } \\
\text { manipulation }\end{array}$ & $\begin{array}{l}\text { Once an attack changes system } \\
\text { process parameters, product quality } \\
\text { assurance is affected by the potential } \\
\text { to bring faulty/harmful products to the } \\
\text { market. }\end{array}$ & $\begin{array}{l}\text { An attacker is manipulating design files } \\
\text { or process parameters to modify the } \\
\text { quality assurance process. }\end{array}$ & $\begin{array}{l}\text { Cyber-physical } \\
\text { system }\end{array}$ & $\begin{array}{l}\text { (Wells, Camelio, } \\
\text { Williams, \& White, } \\
\text { 2014) }\end{array}$ \\
\hline $\begin{array}{l}\text { Intellectual property } \\
\text { theft }\end{array}$ & $\begin{array}{l}\text { Sensitive files such as product designs } \\
\text { and system processes are becoming } \\
\text { comprised of the use of spyware. }\end{array}$ & $\begin{array}{l}\text { Opposing companies/countries using } \\
\text { such compromised information to copy } \\
\text { the competitive edge manufacturing } \\
\text { companies operate. }\end{array}$ & $\begin{array}{l}\text { Cyber espionage, } \\
\text { Spyware, } \\
\text { Data theft }\end{array}$ & $\begin{array}{l}\text { (Friedman, Mack-Crane, } \\
\text { \& Hammond, 2013) }\end{array}$ \\
\hline Cascading failure & $\begin{array}{l}\text { Once one manufacturing system is } \\
\text { compromised, other dependent } \\
\text { systems become } \\
\text { affected/nonoperational such as } \\
\text { delivery systems for the manufactured } \\
\text { products. }\end{array}$ & $\begin{array}{l}\text { Once a hacker can break into a } \\
\text { manufacturing system, other supply chain } \\
\text { components can be damaged. This can } \\
\text { result in product loss or damage. }\end{array}$ & Cascading failure & $\begin{array}{l}\text { (Tang, Jing, He, \& } \\
\text { Stanley, H. E. (2016) }\end{array}$ \\
\hline Supply chain (stuxnet) & $\begin{array}{l}\text { Sensitive intelligence of } \\
\text { manufacturers subcontractors } \\
\text { becomes available for hackers to } \\
\text { understand their system specifications } \\
\text { better, increasing their vulnerability to } \\
\text { attacks. }\end{array}$ & $\begin{array}{l}\text { Finding out who the subcontractors are in } \\
\text { a supply chain to then find vulnerabilities } \\
\text { on a target. }\end{array}$ & $\begin{array}{l}\text { Cyber espionage, } \\
\text { Spyware, } \\
\text { Data theft }\end{array}$ & $\begin{array}{l}\text { (Friedman, Mack-Crane, } \\
\text { \& Hammond, 2013) }\end{array}$ \\
\hline
\end{tabular}




\section{Industry 4.0}

The manufacturing sector is moving to Industry 4.0, and this will bring about entirely new challenges that are introduced due to the complex hyperconnectivity and increased size of the threat landscape. Incorporating the use of Internet of Things (IoT) devices introduces concerns about data loss, theft, privacy, weakened network, and more. With additional devices connected to a network arises more vulnerabilities for hackers to gain access to the said network as additional vectors open up (Copos, Levitt, Bishop \& Rowe, 2016). A home network such as WiFi becoming breached because of an insecure IoT device connected to home WiFi brings up the possibility of data theft and unauthorized network control.

An instance would be an attacker gains control of IoT devices to set wrong data is pulled or pushed from the said device, the actual device functions can be controlled (Bhattacharjee, Salimitari, Chatterjee, Kwiat \& Kamhoua, 2017). An example of this is changing a home's internal temperature reading from an IoT thermostat so that the HVAC system incorrectly heats or cools a home. This can be an annoyance to a degree unless certain items absolutely need to be cooled at all times, such as servers. Letting servers go uncooled can lead to service disruptions. Moreover, with the inclusion of devices such as embedded systems, the complexities continuously rise. This is due to outdated systems and failure to perform path updates and lockdown unnecessary services. In terms of the cybersecurity workforce, there is no move to get talent here as they are going to what is deemed critical or high paying.

\section{Introduction of 5G}

With more data being passed to and from high-tech weaponry devices, there is an increase in lethality (Ullah, Nair, Moore, Nugent, Muschamp \& Cuevas, 2019). For instance, $5 \mathrm{G}$-connected drones can pass and receive more data wirelessly to give them a higher capacity to identify and neutralize targets. Disrupting $5 \mathrm{G}$ radio signals used to communicate between individuals wirelessly is a known vulnerability in $5 \mathrm{G}$ and radio frequency technology in general. The disruption can be done by using a time-select jamming technique to target a specific mobile device. The result is synchronizing the cellular jamming signal to cut the wireless connection to the target device (Lichtman, Rao, Marojevic, Reed \& Jover, 2018).

Tracking unsuspecting user locations via cell tower triangulation can be done using $5 \mathrm{G}$ signals connected to personal mobile devices (Ge, Ye, Yang \& Li, 2016). This is done by gathering data that includes cell tower signal strength from three cell towers to a specific mobile device connected to $5 \mathrm{G}$ - then taking those signal strengths to find and track a user's location. A hacker can start to steal personal information by sniffing a $5 \mathrm{G}$ network to gather and decrypt data packets (Lichtman, Rao, Marojevic, Reed \& Jover, 2018). A technique used to do this is spoofing a cellular base station during the RRC handshake to gather in/outcoming data packets from a cellular device, allowing the hacker to read incoming and outcoming data from mobile devices.

\section{Proposed Solution}

As there are issues with the workforce, which is an essential resource, several tasks need to be automated. To do this, Artificial Intelligence (AI), machine learning, and data science play a pivotal role in securing these critical systems. For a system to connect into a specific sector, it would need to run an AI agent that looks at behavior on the device and captures that data to develop useful and behavioral patterns. Once there is a delineation of what has been baselined as expected. That device is immediately walled off from the network until an additional authentication occurs, 
and a system administrator reviews the logs. Since this would be massive amounts of data, proper data analytics tools would need to be used. From here, a trained data scientist reviews the logs in-depth to validate the actions that have occurred. Once this action has occurred, the system can go back online after it undergoes a quarantine period not to infect the other systems. For the methods that are found infected with malware, then that system would need to be reimaged from a trusted source. The Mission Framework would be something that could be used to ensure that technology, policy, and education are used when developing the solution (Dawson, 2018).

\section{Conclusions}

The protection of these 16 sectors will prove to be a considerable task as the country has inadequately provided numerous sectors only for maintenance. Coupled with technological advances to ease the burden is creating another problem as these systems are becoming weak links for an attack to take advantage of. It is crucial that each of these sectors adopt a cybersecurity framework and set a minimum set of controls that each organization within the industry must agree with. Furthermore, for the integration of newer technologies such as $5 \mathrm{G}$, IoT, and other devices that introduce changes to the networks, it is advised that these systems receive an $\mathrm{AI}$ agent of some sort to monitor device behavior looking for anomalies. Once these anomalies are detected, then that device would require additional authentication measures to remain fully connected. Each year, each sector needs to undergo a check on all systems' security posture to see if their defense in depth strategy is working or if measures need to be immediately taken to mitigate risks that could affect national security or avoid disruption in critical services to the American people.

\section{REFERENCES}

Bhattacharjee, S., Salimitari, M., Chatterjee, M., Kwiat, K., \& Kamhoua, C. (2017, November). Preserving data integrity in iot networks under opportunistic data manipulation. IEEE $15^{\text {th }}$ Intl Conf on Dependable, Autonomic and Secure Computing, 15th Intl Conf on Pervasive Intelligence and Computing, 3rd Intl Conf on Big Data Intelligence and Computing and Cyber Science and Technology Congress (DASC/PiCom/DataCom/CyberSciTech), 446-453.

Bracho, A., Saygin, C., Wan, H., Lee, Y., \& Zarreh, A. (2018). A simulation-based platform for assessing the impact of cyber-threats on smart manufacturing systems. Procedia Manufacturing, Vol. 26, 1116-1127.

Copos, B., Levitt, K., Bishop, M., \& Rowe, J. (2016, May). Is anybody home? Inferring activity from smart home network traffic. IEEE Security and Privacy Workshops $(S P W), 245-251$.

Dawson, M. (2018). Cyber security in industry 4.0: The pitfalls of having hyperconnected systems. Journal of Strategic Management Studies, Vol. 10, Issue 1, 19-28.

Denning, D.E. (2012). Stuxnet: What has changed?. Future Internet, Vol. 4, Issue 3, 672-687.

Department of Homeland Security. (2014). Section 10(a) and 10(b) Report on the United States Coast Guard (USCG) and Maritime Critical Infrastructure Cybersecurity Standards, Section 10(b) Report on the Department of Homeland Security's Chemical Facility Anti-Terrorism Standards (CFATS). Section 10(b) Report on the Transportation Security Administration's (TSA's) Approach to Voluntary Industry Adoption of the NIST. Author. 
Friedman, A.A., Mack-Crane, A., \& Hammond, R.A. (2013). Cyber-enabled Competitive Data Theft: A Framework for Modeling Long-Run Cybersecurity Consequences. Center for Technology Innovation at Brookings.

Ge, X., Ye, J., Yang, Y., \& Li, Q. (2016). User mobility evaluation for 5G small cell networks based on individual mobility model. IEEE Journal on Selected Areas in Communications, Vol. 34, Issue 3, 528-541.

Lewis, J.A. (2002). Assessing the risks of cyber terrorism, cyber war and other cyber threats. Washington, DC: Center for Strategic \& International Studies.

Lewis, B.K. (2012). Social Media and Strategic Communications: Attitudes and perceptions Among College Students (Doctoral Dissertation). ProQuest Dissertations and Thesis Full Texts Database. Oklahoma State University, available at: http://www.prsa.org/Intelligence/PRJournal/Documents/2012LewisNichols.pdf, accessed on 13 September 2020.

Lichtman, M., Rao, R., Marojevic, V., Reed, J., \& Jover, R. P. (2018, May). 5G NR jamming, spoofing, and sniffing: threat assessment and mitigation. IEEE International Conference on Communications Workshops (ICC Workshops), 1-6.

Munro, K. (2012). Deconstructing flame: the limitations of traditional defences. Computer Fraud \& Security, Vol. 10, 8-11.

National Institute of Standards and Technology (NIST). (2014). Framework for Improving Critical Infrastructure Cybersecurity. United States of America.

Obama, B. (2013). Executive order 13636: Improving critical infrastructure cybersecurity. Federal Register, Vol. 78, Issue 33, 11739.

Robles, R.J., Choi, M.K., Cho, E.S., Kim, S.S., Park, G., \& Lee, J. (2008). Common threats and vulnerabilities of critical infrastructures. International Journal of Control and Automation, Vol. 1, Issue 1, 17-22.

Tang, L., Jing, K., He, J., \& Stanley, H. E. (2016). Complex interdependent supply chain networks: Cascading failure and robustness. Physica A: Statistical Mechanics and its Applications, Vol. 443, 58-69.

The White House. (2014). Assessing Cybersecurity Regulations. Available at: http://m.whitehouse.gov/blog/2014/05/22/assessing-cybersecurity-regulations, accessed on 14 September 2020

Ullah, H., Nair, N.G., Moore, A., Nugent, C., Muschamp, P., \& Cuevas, M. (2019). $5 \mathrm{G}$ communication: an overview of vehicle-to-everything, drones, and healthcare use-cases. IEEE Access, Vol. 7, 37251-37268

Wells, L.J., Camelio, J.A., Williams, C.B., \& White, J. (2014). Cyber-physical security challenges in manufacturing systems. Manufacturing Letters, Vol. 2, Issues 2, 74-77.

Wilson, C. (2014). Cyber Threats to Critical Information Infrastructure. Cyberterrorism, 123-136. 